\title{
Effects of a Combined Therapy of ATRA+VEGF on Distal Organs of Emphysematous Mice Model
}

\section{Shivangi Mishra ${ }^{1}$, Megha Neolia ${ }^{1}$, Sherya Patil ${ }^{1}$, Amit Kumar Tyagi ${ }^{2}$ and Jai Prakash Muyal ${ }^{1 *}$}

${ }^{1}$ School of Biotechnology, Gautam Buddha University (GBU), Gautam Budh Nagar, Uttar Pradesh, India

${ }^{2}$ Nuclear Medicine Department, Institute of Nuclear Medicine and Allied Sciences, Defence Research Development Organization, New Delhi, India

*Corresponding Author: Jai Prakash Muyal, School of Biotechnology, Gautam Buddha University (GBU), Gautam Budh Nagar, Uttar Pradesh, India.
Received: August 05, 2020

Published: August 19, 2020

(C) All rights are reserved by Jai Prakash

Muyal., et al.

\begin{abstract}
Pulmonary emphysema is principally associated with the lung. The inflammation is the main cause of emphysema that triggers release of inflammatory mediators. Such inflammatory mediators might get spilled out from the lung to the proximal and distal organs, hence may affects other organ's functioning as well. In this light, firstly, we attempted to determine the spillage of inflammatory mediators on liver and heart organs of emphysematous lung mice model. Secondly, to elucidate the beneficial role of ATRA+VEGF supplementation towards such spillage of inflammatory mediators by studying the antioxidant levels, which leads in tissue damage and hence affects its function.

To analyse these two objectives, in silico study has been performed for the prediction of the gene interaction network between elastase, ATRA, and VEGF, respectively with extracellular regulated kinases (ERK) pathway. Furthermore, antioxidant levels and histopathology were studied in before elastase-treatment, after elastase-treatment and after elastase-treated group received ATRA+VEGF conditions using liver and heart tissues.

Elastase-treated group received ATRA+VEGF has proven to be beneficial in reducing inflammation as the levels of anti-oxidants was significantly reduced. Moreover, the tissue architecture of liver and heart was improved due to the ATRA+VEGF supplementation. At last, gene interaction network results provide a promising role of ATRA and VEGF in rectification of inflammation via involvement of spilled out inflammatory mediators. However, an elaborate experimental study is further required to investigate the beneficial role of ATRA+VEGF therapy on ERK pathway towards amelioration of inflammation.
\end{abstract}

Keywords: ATRA+VEGF Therapy; Organs; Chronic Obstructive Pulmonary Disease (COPD)

\section{Introduction}

Chronic Obstructive Pulmonary Disease (COPD) is one of the long-term lung condition caused by the lung irritants i.e. environmental pollutants and cigarette smoking. It is characterized by the poor passage of the air flow through lungs. Persistent inflammation in the lungs damages the air spaces, makes it enlarged hence strengthens the disease progression. Two of the chronic pathological conditions are known to made up for COPD conditions i.e. chronic bronchitis and emphysema. Chronic bronchitis is chronic lung damage due to irritation and inflammation of the bronchial tubes. The irritation in the tubes builds up the mucus and hence the tubes are not able to carry the air to and from the air sacs in 
the lungs properly. This condition damages the alveoli and makes it difficult to breathe. Whereas, emphysema is characterized by the proteolytic destruction of alveolar spaces, enlarged air spaces in turn leading to shortness of breath, wheezing, cough, mucus accumulation and fatigue $[1,2]$. The pathogenesis of emphysema chiefly involves chronic inflammation, which arises due to the continuous exposure to cigarette smoke or to long-term exposure to toxic gases and particles, due to which a rapid induction of innate immunity could occur. Such conditions in the lung may recruit a large influx of neutrophils into the airways, which further may increase the levels of oxidant-antioxidant imbalance, protease-antiprotease imbalance, ineffective repair, and apoptosis, leading to the lung tissue remodelling [3]. Such inflammatory mediators generated during emphysematous condition are thought to be permeated into the circulatory system, leading to systemic inflammation and thereby affecting other vital organs and their function [4]. Moreover, a little information is available regarding the comorbidities due to "spill over" of inflammatory mediators in case of emphysema patients. However, it is evident that there is systemic oxidative stress, activated circulating inflammatory cells and increased pro-inflammatory cytokines such as TNF- $\alpha$, C-reactive protein (CRP), IL-6, fibrinogen, activated leucocytes in plasma in case of COPD [5-8]. The effects of the spillage of these inflammatory mediators might affect the circulatory and hepatic systems; however, no data is reported in this direction if line so far.

Alveolar epithelium tissue regeneration is the only hope to cure emphysema, nevertheless, until today there is no treatment option is available for emphysema. Therefore, an urgent requirement for a development of therapy option against is required. Recently only, All-trans retinoic acid (ATRA), which is the biologically active metabolite of vitamin-A, possesses anti-inflammatory property have been found to play a crucial role in an alveolar epithelial cell proliferation, differentiation and its survival [9]. Additionally, in the lung, vascular endothelial growth factor (VEGF) role has been found as a potential candidate towards alveolar maintenance and repair processes [10]. The vast pulmonary capillary bed is crucial in allowing efficient gas exchange to occur between the alveoli and circulating blood supply. Interestingly, VEGF signalling has been found to play a pivotal role during airway growth as the lung progressively acquires a rich blood supply through the growth of endothelial cells in the pulmonary mesenchyme, which is crucial for the gas exchange function of the human lung. Injury and loss of this tissue contributes to the pathology of many forms of lung [11]. However, the potential role of a combined therapy of ATRA and VEGF in reduction of spillage of inflammatory mediators to other organs like heart and liver has not yet been clearly explored. Therefore, in this study we tried an attempt to evaluate the beneficial role of ATRA+VEGF therapy on heart and liver tissue and their respective physiological functions in an established pulmonary emphysema rat model. However, the exogenously given elastase was aimed to cause pulmonary emphysema; it may have affected heart and liver's physiological functions because of the spill over of inflammatory mediators (As shown in figure 1).

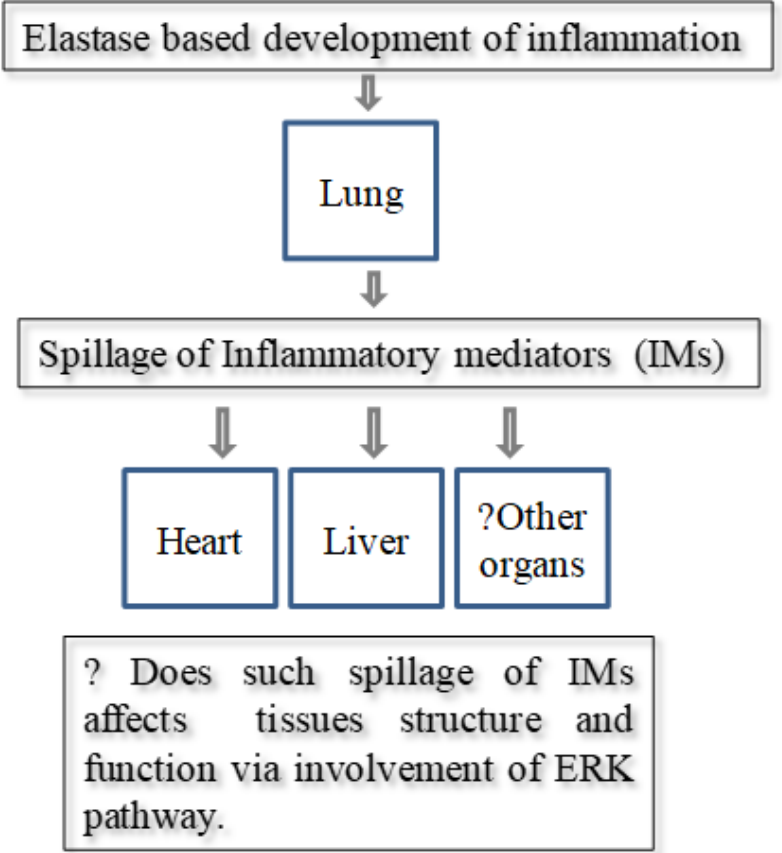

Figure 1: A schematic representation of the current study's proposal. Porcine pancreatic elastase has a tremendous tendency towards development of chronic inflammation in the lungs of mice. There might be chances of spill-over into the systemic circulation and infiltrates into the liver, heart and other organs leading to inflammation in liver and heart. The spill-over of inflammatory mediators may also encourage in dysregulation of physiological processes of the liver and heart tissues. 


\section{Material and Methods}

Preparation of animal models

All experimental models were prepared according to the procedure and dosing described by Seifart C., et al. [12] and were in compliance with National and International guidelines approved by the regional government. The models were prepared at Institute of Nuclear Medicine and Allied Sciences, DRDO, New Delhi under the supervision of Dr. Amit Kumar Tyagi. Pathogen-free six weeks old C57BL male mice at a body weight of about $20 \mathrm{~g}$ were randomly assigned to three different experimental models ( $\mathrm{n}=$ 5 per group). An experimental animal model was created as per following: Group 1 (emphysema model): Development of animal model of pulmonary emphysema by Porcine Pancreatic Elastase (PPE). The PPE induced Lung emphysema is the oldest and one of the best characterized animal model, which shows similar histological changes that appeared in emphysema in humans by mean of long exposure of cigarette- based model $[13,14]$. Emphysema was induced in mice's lungs $(n=5)$ by single instillation of $25 \mathrm{U} /$ $\mathrm{kg}$ b.w. PPE via oropharyngeal method on two different occasion i.e. at day 1 and day 11. From at day 21 to 33, each day PPE treated lungs of animals received a single bolus of $100 \mu \mathrm{l}$ of saline (subcutaneously). Group 2 (therapy model): To test for the therapeutic effect of ATRA+VEGF, emphysema was induced as per described in group 1. From at day 21 to 33, each day PPE treated lungs (n = 5) received ATRA and VEGF, which was dissolved in saline in a final volume of $100 \mu \mathrm{l}$ of ATRA+VEGF (subcutaneously). Group 3 (Healthy model): At day 1 , animals $(n=5)$ received a single bolus of $150 \mu \mathrm{l}$ saline (PBS) by oropharyngeal and second bolus on day 11 . From at day 21 to 33, each day animals received a second bolus of $150 \mu \mathrm{l}$ of saline (subcutaneously). Animals were sacrificed on day 34 by cervical dislocation. Along with the lung tissue, heart and liver organs were harvested and were stored at $-80^{\circ} \mathrm{C}$.

\section{Gene interaction}

The phenomenon of two or more genes affecting the expression of each other in various ways in the development of a single character of an organism is known as gene interaction. This gene interaction can be analysed by various available online tools. The test gene, which is elane (a protein called neutrophil elastase); NCF1, an ATRA responsive gene; and Kdr, CD34, Flt1A are the VEGF marker genes; Myh6, Myb1, a heart genes; Notch 1, Nfe212, a liver genes, respectively, were given as input for the prediction of their interaction with the candidate genes of ERK-pathway by using online free available programme that is called GeneMANIA. The outcome of this programme may help in dissecting their co-expression, physical interactions, shared protein domains and co-localization.

\section{Lung fixation and histopathology}

Heart and Liver tissues were fixed in $6 \%$ phosphate-buffered paraformaldehyde and stored in the refrigerator $\left(4^{\circ} \mathrm{C}\right)$. Tissue blocks were prepared using molten paraffin and allowed to cool and solidify before making tissue sections. 5-10 $\mu \mathrm{m}$ thin tissue sections were cut using a microtome (Spencers rotary microtome, India). Subsequently, tissue sections were deparaffinised three times by Xylene, rehydrated with different concentrations of ethanol and stained with hematoxylin and eosin (H\&E) stain.

Biochemical analysis for catalase (CAT) and glutathione-stransferase (GST)

Heart and liver tissues were homogenized separately in phosphate buffer ( $\mathrm{pH} 7.4$ ) and centrifuged at $12,000 \times \mathrm{g}$ at $4^{\circ} \mathrm{C}$ for 30 min to get tissue homogenates which were further used for antioxidant activity determination. Tissue antioxidant status was determined by estimation of antioxidant activities of catalase (CAT) as described by Maehly., et al. [15]. Activity of glutathione-S-transferase was done as described by Mohandas., et al [16].

Statistical analysis: Differences between experimental groups were tested for significance using Student's unpaired t-test used to determine the level of significance of differences between control versus emphysema and emphysema versus ATRA+VEGF therapy, respectively using GraphPad Prism version 5, San Diego, USA. Levels of significance are indicated by ${ }^{*}=p<0.05$.

\section{Results}

In-silico study on effects of elastase, ATRA and VEGF on ERK pathway

In the current study, a gene interaction has been done by using GeneMANIA online programme. The results obtained from GeneMANIA show the interactions between test genes with the genes of ERK-pathway (Figure 2A-F). The elane gene has been found in affecting the candidate genes of ERK pathway, indirectly, which also showing an interaction with the liver genes (Notch1 and Nfe212; figure 2A). NCF1, which is the ATRA responsive gene has shown its interaction with the genes of ERK pathway (figure 2B). Further- 
more, the VEGF responsive genes that are Kdr, Flt1, Angpt1, Pecam and CD34 have shown a strong interaction network with the genes of ERK pathway and liver's gene (Figure 2C). On the other hand, a strong interaction network of elane gene, NCF1 (ATRA responsive gene) and marker genes of VEGF with ERK pathway genes were noticed using heart's marker genes i.e. Myh6 and Myb1 (Figure 2D-2F).
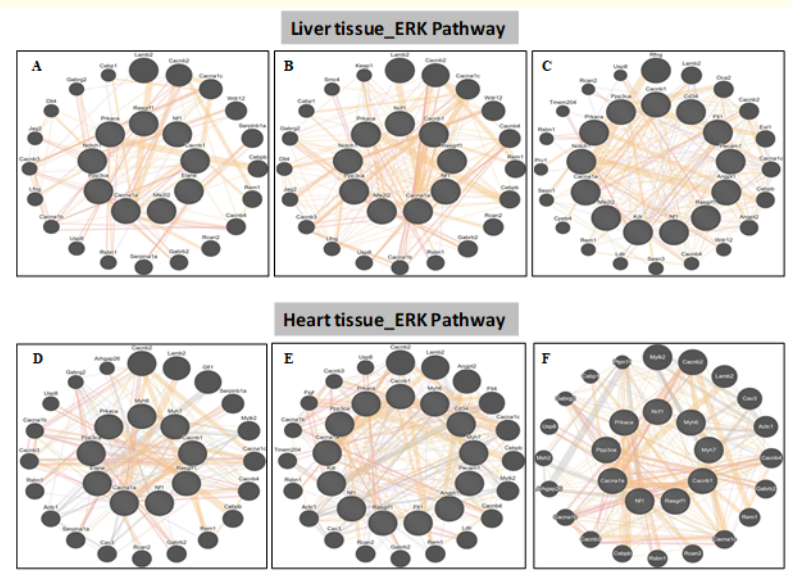

Figure 2: Gene interaction network study was performed using marker genes of Elastase, ATRA and VEGF using liver and heart marker genes. The marker genes of Elastase, ATRA and VEGF has shown a strong interaction with the genes of ERK pathways, as indicate with the strong lines, showing involvement of elastase in induction of inflammation while a combination of ATRA and VEGF might be are one of the candidates for attenuation of such induced inflammation due to elastase based spill-over of IMs.

Effects of elastase and ATRA+VEGF therapy on antioxidant activity

Changes in liver and heart activities of antioxidant enzymes (i.e. CAT and GPx) in all the experimental groups of the rat were obtained. With the liver and heart tissue, elastase has shown its significant effect by showing in an induction of the activities of CAT and GPx as compared to the healthy group (Figure 3A-3D). Particularly in liver tissue, treatments with ATRA+VEGF markedly improved the effects of elastase and restored the activities of CAT and
GPx in the ATRA+VEGF therapy group and were comparable to the healthy group (Figure 3C and 3D).
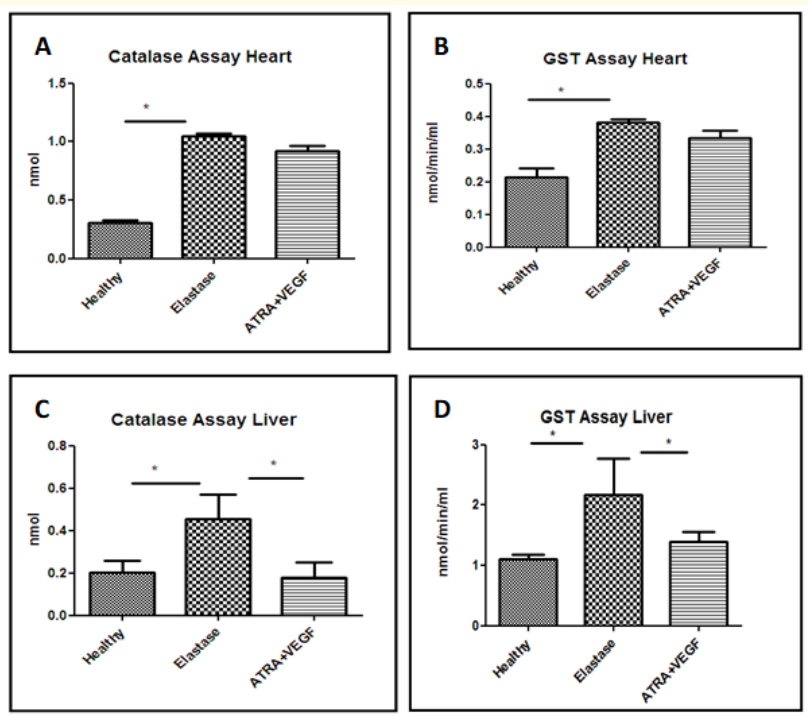

Figure 3: Effects of elastase and ATRA+VEGF on anti-oxidant level: Significant induction in the levels of CAT and GPx was noticed in elastase group against to healthy group for both the organs that is liver and heart. However, in case of liver tissue, upon ATRA+VEGF supplementation to elastase treated group, a significant reduction of CAT and GPx levels was observed and interestingly are well comparable to healthy group. Graphs indicate mean values with standard deviation. Representative graphs of pooled tissue homogenate samples $(n=3)$ from each group in triplicates. Data were analysed by means of unpaired

t-test to test for the effect of ATRA+VEGF and elastase, respectively. ${ }^{*} \mathrm{p}<0.05$.

Effects of elastase and ATRA+VEGF therapy on liver and heart tissue's architecture

The healthy group showed the structure of the liver and heart tissues under normal physiological conditions (Figure 4A and 4D). In liver tissue, it clearly shows the portal vein, hepatic artery, lobule boundary and the connective tissue within the liver. No enlarged spaces, dilated air spaces were seen (Figure $4 \mathrm{~A}$ ), however, the effect of elastase can be seen, the histopathological based observation reveals the number of kupffer cells increased in the elastase 
treated group than the healthy group. Moreover, the lesion slightly compresses the liver tissue. Also there is no capsule around the lesion and other follicles are normal as they indicate the lymphocytes. These changes in the structure of liver tissue are associated with some inflammatory conditions happening in the body (Figure 4B). We saw slender improvement in the healing of tissue upon supplementation of ATRA+VEGF on liver tissue treated with elastase (Figure 4C). On the other hand, heart tissue which was obtained from the elastase treated lungs has shown less intercalated disc, less number of cardiac muscle nuclei and disrupted fibres than heart tissue obtained from healthy lungs (Figure 4E). Interestingly, the beneficial role of ATRA+VEGF therapy on heart tissue obtained from the elastase treated ATRA+VEGF was seen by recording more number of intercalated discs, more number of muscle nuclei and defined stratified muscle fibres than heart tissue of elastase treated lung's group (Figure 4F).

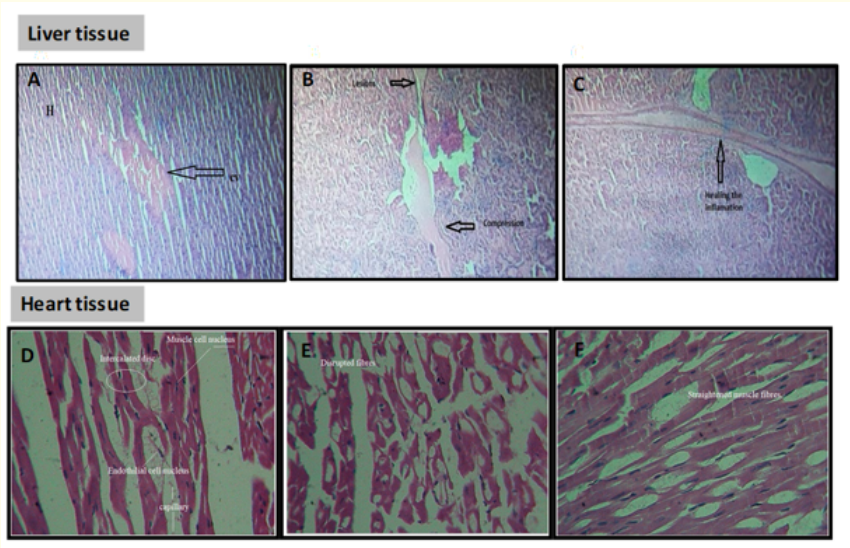

Figure 4: Histopathology of liver and heart tissue: Hematoxylin and Eosin staining of liver and heart tissue sections show normal histology in healthy group, the number of kupffer cells increased, the lesion slightly compresses and no capsule around the lesion and other follicles in liver tissue of elastase treated group (Figure 4B) while in case of heart tissue, a less intercalated disc, less number of cardiac muscle nuclei and disrupted fibres were seen (Figure 4E). Interestingly, ATRA+VEGF therapy restored all these changes and able to bring them closer to the healthy group (Figure 4D,F). All micrographs were captured at identical magnification (40X). From each liver and heart specimen (total 3), an average of 3 different sections was used, and in the sections generally, 3 representative non-overlapping fields were selected.

\section{Discussion}

This study has shown the effect of ATRA+VEGF therapy on the liver and heart tissues of elastase induced emphysematous mice. It is evident from previous studies that COPD involves inflammatory spillage [17-20]. The hypothesis of the present study was that, this might be possible that at the time of inflammation because of elastase given into the lung may contribute into the spill over of inflammatory mediators from lung into the liver, heart and other organs via the circulatory process. Such spillage of inflammatory mediators might possibly be involved in the dsyregulation of metabolic and physiological processes occurring in liver tissue and heart tissue. By knowing spill over of inflammatory mediators and its consequences to the other organs may be helpful in dissecting medical conditions, if arise.

It has been reported that the increased inflammatory response to be mediated indirectly via the activation of mitogen-activated protein (MAP) kinase [21] and extracellular regulated kinases (ERK) pathways, which are mainly involved in regulating the expression of several inflammatory genes [22]. ERK pathway is chiefly involved in cell proliferation and also involved in several inflammatory processes [23]. There are several reports, where authors have suggested that the ERK pathway was deregulated in emphysematous lung condition $[24,25]$. Hence, the foremost objective of this current study is to examine whether ATRA+VEGF therapy has a possibility towards amelioration of such dsyregulation of ERK pathway of liver and heart tissues due to elastase? To validate this hypothesis, a combined form of ATRA+VEGF was given intraperitoneally to the elastase treated mice. To observe ATRA+VEGF therapy's potential effect, histopathology of liver and heart tissues were performed, the anti-oxidant levels were also determined. Moreover, it is also important to investigate the interaction of elastase, ATRA and VEGF with the ERK pathway, which gets generally hyper during the time of inflammation, which was studied here using insilico approach.

Our in-silico results show an interaction between the test genes (elastase, ATRA and VEGF) with the ERK pathway. Such interaction provide us a hint that there might be a possibility that exogenously given elastase to the lungs of mice may not only cause lung damage but also a liver and heart tissue, provided if an inflammatory mediators gets spilled out from the lungs to the other vital organs. Nevertheless, an interaction of ATRA and VEGF which was given in combination during animal model preparation has also shown 
a fair interaction of ATRA and VEGF marker genes with the ERK pathway. These results further provide us a hint that such combination may be beneficial in reverting ERK pathway if gets deregulated due to the inflammation by mean of elan gene here (Figure 2, $\mathrm{B}, \mathrm{C}$ and $\mathrm{E}, \mathrm{F}$ ).

Under physiological situation a balance exists between the quantity of reactive oxygen species produced in normal cellular metabolism and the endogenous antioxidant defence. At the time of acute inflammation, there is activation of leukocytes and neutrophils, which release reactive oxygen species that eventually leads to oxidative stress. To balance them, anti-oxidants are required in protecting the tissues from the oxidative damage. Yet again, a positive signature of ATRA+VEGF therapy has been noticed in liver tissue of elastase treated lungs group, showing in reduction in the activities of glutathione peroxidase (GPx) and catalase (CAT) in comparison to the elastase treated lungs group. Infiltration of leukocytes cells from circulatory system is essential during inflammatory response. The inflammatory response is the coordinate activation of signaling pathways that regulate inflammatory mediator levels in resident tissue cells and inflammatory cells recruited from the blood. Here, an induction in the anti-oxidant levels (Figure $3 A-D$ ) might be due to the migration of inflammatory mediators from lung tissue to the heart and liver tissues. Such migration of inflammatory mediators may activate the anti-oxidants towards minimisation of ROS, so that it may protect the tissue of concern. Moreover, a CAT and GPx activity were recorded high in COPD patients and is directly proportional to the severity of the disease [26]. Interestingly, we found a potential role of ATRA+VEGF therapy in maintaining a balance between oxidants and antioxidants for proper functioning of any tissue.

Since the anti-oxidant levels were induced due to the elastase based infiltration of inflammatory mediators it is also now become one of the concerns to further investigate its impact on tissue structures of liver and heart. Therefore, we have performed histopathology of liver and heart tissues. In both tissues, we have found a change in the structural architecture than healthy tissue. A fewer numbers of hepatocytes with a compression in the liver tissue were seen in the liver tissue of emphysematous lungs model than healthy liver (Figure 4B). However, healing and increase in the hepatocytes number was observed after ATRA+VEGF therapy in the liver of elastase treated emphysematous model (Figure 4c).
Since ATRA has an anti-inflammatory effect, and thus might be responsible here in reducing ongoing inflammation while VEGF helps in growth of endothelial cell and in inducing in the proliferation of hepatic sinusoidal cells [27] which contributing in maintaining a capillary bed, which is crucial for efficient gas exchange for the function of the liver. Whereas in case of heart tissue, disrupted cardiac fibres, less number of muscle cell nucleus and endothelial cell nucleus was noticed in elastase induced emphysema model than healthy ones (Figure 4E). Once again, ATRA+VEGF therapy has shown its beneficial effect towards reduction of ongoing inflammation and in induction of endothelial cell and cardiac muscle cell nucleus of the heart tissue, obtained from elastase treated emphysema model (Figure 4E). Moreover, our finding is also in accordance to the study conducted by Drowley., et al. they have shown that ATRA has a tendency towards an increase of the number of cardiomyocytes, endothelial cells, smooth muscle cells, cardiac fibroblasts [28].

\section{Conclusion}

The current study focused on the inflammatory and anti-inflammatory effects of elastase and a combined therapy of ATRA+VEGF on the liver and heart tissues of elastase treated lung mice model. Our in silico findings provide us a hint that elastase, ATRA and VEGF has an affinity for its interaction with ERK pathway. Secondly, the histopathology images of liver and heart tissues show degeneracy in tissue architecture in elastase treated group, however, which were attenuated by providing ATRA+VEGF therapy. The spills out inflammatory mediators play a crucial role in induction of CAT and GPx levels in liver and heart tissues, nevertheless, CAT level was reduced significantly in liver tissue of elastase treated lungs received ATRA+VEGF. Thus this preliminary data suggesting that elastase has a potential role towards infiltration of inflammatory mediators from one organ to another while a combined therapy of ATRA+VEGF has a therapeutic potential in correction of those possible damages. However, an elaborate studies need to be further conducted to explore the regeneration mechanism.

\section{Acknowledgment}

The authors thank Dr. Amit Tyagi (Institute of Nuclear Medicine and Allied Sciences, DRDO, New Delhi, India) for providing mice and animal facility. The authors are thankful to Mr. Basav Hazarika (SoBT, GBU, Greater Noida, India) for helping in refer- 
ence maintenance. Special acknowledgement to the SCIENCE and ENGINEERING RESEARCH BOARD [SERB]-Department of Science and Technology, Government of India for providing funds. FILE NO.EEO/2018/000050.

\section{Conflict of Interest}

None.

\section{Bibliography}

1. S Uniyal., et al. "ATRA reduces inflammation and improves alveolar epithelium regeneration in emphysematous rat lung". Biomedicine and Pharmacotherapy 108 (2018): 1435-1450.

2. M King Han., et al. "Chronic Obstructive Pulmonary Disease: Definition, Clinical Manifestations, Diagnosis, and Staging". UpToDate (2016).

3. A Sharafkhaneh., et al. "Pathogenesis of emphysema: From the bench to the bedside". Proceedings of the American Thoracic Society 5.4 (2008): 475-477.

4. PJ Barnes and BR Celli. "Systemic manifestations and comorbidities of COPD". European Respiratory Journal. 33.5 (2009): 1165-1185.

5. AGNAA Noguera., et al. "Enhanced neutrophil response in chronic obstructive pulmonary disease". Thorax 56 (2001): 432-437.

6. WQ Gan., et al. "Association between chronic obstructive pulmonary disease and systemic inflammation: A systematic review and a meta-analysis". Thorax 59.7 (2004): 574-580.

7. P Kirkham and I Rahman. "Oxidative stress in asthma and COPD: Antioxidants as a therapeutic strategy". Pharmacology and Therapeutics 111.2 (2006): 476-494.

8. AMWJ Schols., et al. "Evidence for a relation between metabolic derangements and increased levels of inflammatory mediators in a subgroup of patients with chronic obstructive pulmonary disease". Thorax 51.8 (1996): 819-824.

9. G De Carlo Massaro and D Massaro. "Retinoic acid treatment abrogates elastase-induced pulmonary emphysema in rats". Nature Medicine 3.6 (1997): 675-677.

10. G NEUFELD., et al. "Vascular endothelial growth factor (VEGF) and its receptors". FASEB Journal 13.1 (1999): 9-22.
11. KH. "Role of vascular endothelial growth factor in the pathogenesis of chronic obstructive pulmonary disease". Medical Science Monitor 13.11 (2007): 189-195.

12. C Seifart., et al. "All-trans retinoic acid results in irregular repair of septa and fails to inhibit proinflammatory macrophages". European Respiratory Journal 38.2 (2011): 425-439.

13. R Rodrigues., et al. "A murine model of elastase- and cigarette smoke-induced emphysema TT - Modelo murino de enfisema induzido por instilação de elastase e exposição a fumaça de cigarro". Jornal Brasileiro de Pneumologia 43.2 (2017): 95-100.

14. R Rodrigues., et al. "An experimental model of elastase and cigarette smoke- induced emphysema in mice". European Respiratory Journal 44.58 (2014).

15. AC MAEHLY and B CHANCE. "The assay of catalases and peroxidases". Methods of Biochemical Analysis 1 (1954): 357-424.

16. TD Mohandas J., et al. "Differential distribution of glutathione and glutathione-related enzymes in rabbit kidney. Possible implications in analgesic nephropathy". Biochemical Pharmacology 33.11 (1984): 1801-1807.

17. N J Sinden and R A Stockley. "Systemic inflammation and comorbidity in COPD: A result of 'overspill' of inflammatory mediators from the lungs? Review of the evidence". Thorax 65.10 (2010): 930-936.

18. R Tkacova., et al. "Metabolic phenotype and adipose tissue inflammation in patients with chronic obstructive pulmonary disease". Mediators of Inflammation (2010).

19. J Lee., et al. "Cigarette Smoking and Inflammation". Journal of Dental Research 91.2 (2012): 142-149.

20. S Uniyal., et al. "ATRA modulates gluconeogenesis and triglyceride biosynthesis pathway of liver in elastase induced emphysematous rats". Journal of Experimental Animal Science 2.2 (2017): 109-125.

21. W Ziaziaris and L Munoz. "Mitogen-activated protein kinase-activated protein kinase 2 in neuroinflammation, heat shock protein 27 phosphorylation, and cell cycle: Role and targeting fadi maged shokry gurgis". Molecular Pharmacology 85.2 (2014): 345-356. 
22. H K Kim. "Role of ERK/MAPK signalling pathway in anti-inflammatory effects of Ecklonia cava in activated human mast cell line-1 cells". Asian Pacific Journal of Tropical Medicine 7.9 (2014): 703-708.

23. JD Becky A., et al. "Extracellular Regulated Kinase/Mitogen Activated Protein Kinase Is Up-regulated in Pulmonary Emphysema and Mediates Matrix Metalloproteinase-1 Induction by Cigarette Smoke". Journal of Biological Chemistry 279.17 (2004): 17690-17696.

24. J P Muyal., et al. "Effect of recombinant human keratinocyte growth factor in inducing Ras-Raf-Erk pathway-mediated cell proliferation in emphysematous mice lung". Inhalation Toxicology 26.13 (2014): 761-771.

25. JC., et al. "Crucial requirement of ERK/MAPK signaling in respiratory tract development". Development 141 (2014): 31973211.

26. S Singh., et al. "Evaluation of Oxidative Stress and Antioxidant Status in Chronic Obstructive Pulmonary Disease". Scandinavian Journal of Immunology 85.2 (2017): 130-137..

27. H Shimizu., et al. "Vascular endothelial growth factor secreted by replicating hepatocytes induces sinusoidal endothelial cell proliferation during regeneration after partial hepatectomy in rats". Journal of Hepatology 34.5 (2001): 683-689.

28. L Drowley., et al. "Discovery of retinoic acid receptor agonists as proliferators of cardiac progenitor cells through a phenotypic screening approach". Stem Cells Translational Medicine 9.1 (2020): 47-60.

\section{Assets from publication with us}

- Prompt Acknowledgement after receiving the article

- Thorough Double blinded peer review

- Rapid Publication

- Issue of Publication Certificate

- High visibility of your Published work

Website: www.actascientific.com/

Submit Article: www.actascientific.com/submission.php

Email us: editor@actascientific.com

Contact us: +919182824667 